\title{
THE EFFECT OF GURNEY FLAP ON FLOW CHARACTERISTICS OF VERTICAL AXIS WIND TURBINE
}

\author{
TAURISTA PERDANA SYAWITRI ${ }^{1,2}$, YUFENG YAO $^{1, *}, \mathrm{JUN}$ YAO $^{3}$ AND BUDI CHANDRA $^{1}$ \\ ${ }^{I}$ Department of Engineering, Design and Mathematics, University of the West of England, Bristol, BS16 1QY, \\ United Kingdom \\ ${ }^{2}$ Department of Mechanical Engineering, Universitas Muhammadiyah Surakarta, Surakarta, 57162, Central \\ Java, Indonesia \\ ${ }^{3}$ School of Engineering, College of Science, University of Lincoln, Brayford, Lincoln, LN6 7TS, United \\ Kingdom \\ *Correspondence:Yufeng.Yao@uwe.ac.uk
}

\begin{abstract}
Recently, Gurney Flap (GF) has been applied in Horizontal Axis Wind Turbine (HAWT) for performance increase by enhancing lift coefficient. Compared to HAWT, the research on GF application for Vertical Axis Wind Turbine (VAWT) is very limited. Moreover, most work evaluated a GF geometry attached to the trailing-edge of a stationary airfoil without the rotating effects as experienced in VAWT scenario. For this reason, a three-straight-bladed VAWT rotating blades with GF is studied by the solution of transient RANS equations together with stress-blended eddy simulation (SBES) turbulence model to investigate the GF height effect and the flow characteristics near the blade trailing-edge. The results have shown that by introducing the rotating, an optimum GF height is found to be $3 \%$ of the airfoil chord, slightly higher than $2 \%$ chord in a stationary airfoil case. In addition, the addition of GF can suppress deep stall of VAWT blades, thus eliminating negative instantaneous moment coefficient and improving the turbine performance.
\end{abstract}

Keywords: Stress-blended eddy simulation; Vertical axis wind turbine; Gurney flap; Delayed flow separation.

\section{Introduction}

There are increased researches for Vertical Axis Wind Turbines (VAWTs) recently due to its outperformance in urban area applications than Horizontal Axis Wind Turbines (HAWTs). ${ }^{1}$ However, VAWTs still need to improve its efficiency and self-starting ability, to compete with HAWTs commercially. One possible solution is to introduce a passive device such as Gurney Flap (GF) at the blade trailing-edge to enhance the blade lift coefficient. For a stationary single airfoil, the existence of GF will generate counterrotating vortices downstream of the flap along with a significant flow turning over leeward of the flap ${ }^{2}$, leading to the decreases of wake momentum deficit and the increases of lift force. Recently, GF has been applied in HAWTs to improve the performance of turbine blades with smaller chord length. ${ }^{3}$ The results showed that blade with GF indeed can increase the power generation of the wind turbine compared to those with no GF installed. In case of VAWTs, there are very limited studies on the GF application. ${ }^{4-5}$ Mostly, 
researches only presented the outcome of power coefficient increment (by referring to the work of Liebeck ${ }^{2}$ ) but with no any further analyzing and explaining on its underlying flow physics and characteristics.Moreover, previous work did not consider the rotating effect that could have significant impact on blade-wake interaction and power production of VAWT due to unsteady power conversion at high angle of attack (AoA) in a dynamic stall regime. ${ }^{6}$ The rotating blade will also affect the optimization of GF geometries at different AoA. ${ }^{7}$ Hence, the optimum GF geometries obtained from a stationary single airfoil is not viable for the application of VAWTs. Therefore, this study intends to investigate the effect of GF height on the performance of a three-straight-bladed VAWT, and to realise its underlying flow physics and characteristics.

\section{Methodology}

Computational Fluid Dynamics (CFD) are used to solve the unsteady flow around a two-dimensional model of a rotating three-straight-bladed VAWT with NACA0021 airfoil profile featured in the study of Castelli et al. ${ }^{8}$ The Gurney Flap is mounted in the trailing edge of the airfoil. GF width is fixed at $0.33 \%$ of the airfoil chord $(c)$. The height of GF is varied from $1 \%, 1.5 \%, 2 \%, 3 \%$ and $4 \%$ of $c$, measured from the lower point of the trailing edge wall. The free stream velocity is set to be $9 \mathrm{~m} / \mathrm{s}$. This study is conducted by an optimum tip speed ratio (TSR) of an original airfoil without GF (i.e. $T S R=2.64)$. The simulation is carried out with pressure-based solver due to low-speed incoming flow. After preliminary study, a stress-blended eddy simulation (SBES) turbulence model with transition shear-stress transport (SST) is chosen as it gave the smallest deviations compared to the experimental results. ${ }^{8}$

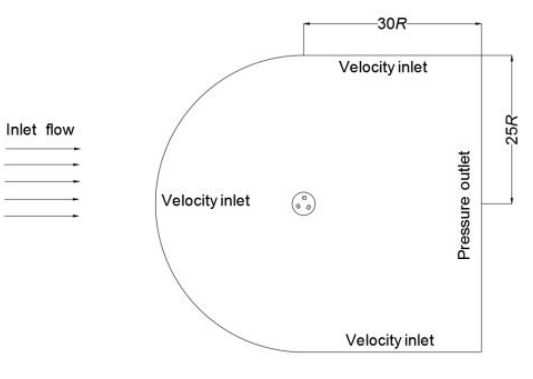

(a) Overall Computational Domain

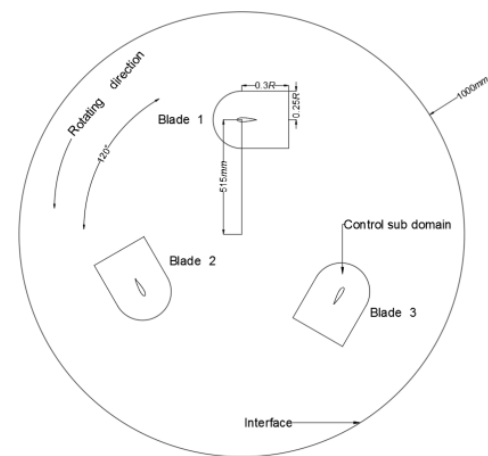

(b) Rotating core sub-domain

Fig. 1. Detailed computational domain and sub-domains of the simulation.

A C-type grid topology is used and the computational domain is divided into three subdomains (see Fig. 1), namely far field (non-rotating domain), rotating core sub-domain (rotating domain) and three control sub-domain with embedded blades. The first grid height on blade surface is designed to satisfy the criteria of non-dimensional wall distance $\Delta y_{1}{ }^{+}<$ 1 for transition SST turbulence model. A grid convergence study is conducted and it is found that a total number of 189,807 grids with 174 cells around one blade surface is able to produce CFD predictions in good agreement with available experimental results. ${ }^{8}$ 


\section{Results and Discussion}

Fig. 2 shows that in general the power coefficient $\left(C_{p}\right)$ of VAWT will increase with the increase of the Gurney Flap height, with a maximum $C_{p}$ value achieved at a height of about $3 \%$ of the chord. After that, the GF will lose its capability to further increase the $C_{p}$. Compared to single stationary airfoil of optimum GF height of $2 \%$ of $c$, this optimum GF height is slightly higher, possible due to rotating effect and wake-blade interactions. This confirms that previous findings from single stationary airfoil with GF is not applicable to the rotating wind turbine blade applications.

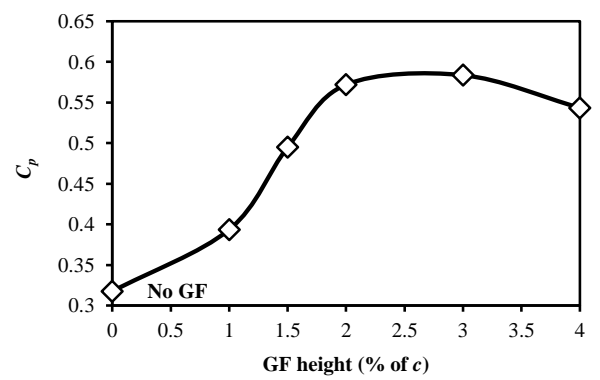

Fig. 2. Comparison of power coefficient of VAWT with and without GF at various GF heights.

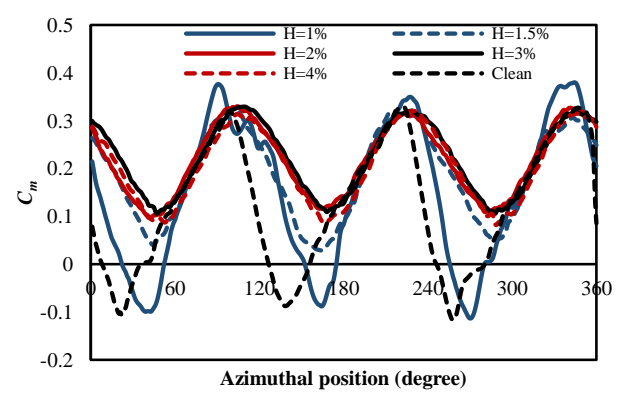

Fig. 3. Comparison of instantaneous total moment coefficients distribution of VAWT with and without GF at various GF heights.

Fig. 3 illustrates the instantaneous moment coefficients $\left(C_{m}\right)$ distribution over one turbine revolution at different GF heights. The $C_{m}$ distribution demonstrates that the addition of GF with a height equal or higher than $1.5 \%$ of airfoil chord can remove the negative value of $C_{m}$, resulting in the increment of power generation of the turbine. Moreover, the presence of GF can postpone the deep stall of turbine blades, indicated by slower drop rate in $C_{m}$ curves after reaching its maximum peak.

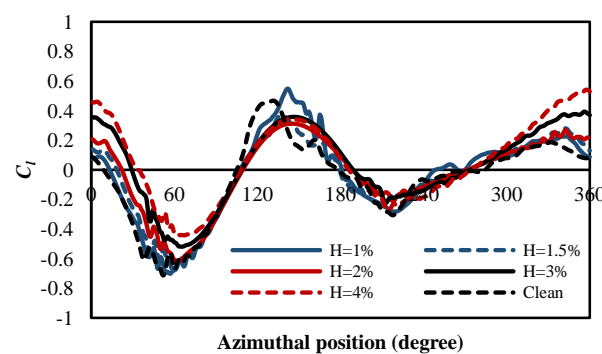

Fig. 4. Comparison of instantaneous lift coefficients distribution of one rotation cycle at various GF heights.

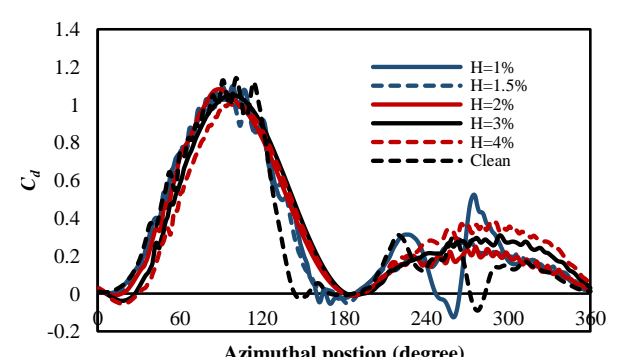

Fig. 5. Comparison of instantaneous drag coefficients distribution of one rotation cycle at various GF heights.

In order to further understand the effect of GF addition on performance, distributions of instantaneous lift coefficients $\left(C_{l}\right)$ and drag coefficients $\left(C_{d}\right)$ of one blade over one turbine rotation cycle are depicted in Fig. 4 and Fig. 5, respectively. It shows that the GF addition can reduce the fluctuation amplitude of $C_{l}$ and delay the sudden increase of $C_{d}$ at an azimuthal position of about 90 degrees. This confirms that GF can ease the deep stall of turbine blades. The unsteady behaviour of $C_{l}$ and the rapid increment of $C_{d}$ suggests that 
the VAWT starts to experience the stall between azimuthal positions of 60-100 degrees, approximately. ${ }^{7}$ Further investigation on the pathlines coloured by vorticity magnitude shows that the addition of GF can certainly introduce vortices in downstream of GF which can lessen the adverse pressure gradient near the trailing edge. Moreover, the contour of root mean square error (RSME) velocity magnitude suggests that GF can enhance the velocity over the suction surface side while the velocity decreases on the pressure surface side. As a result, the flow separation can be delayed and the total circulation of airfoil increases. Therefore, lift enhancement which leads to power generation increment can be achieved by using GF.

\section{Conclusion}

The effect of GF at blade trailing edge and its optimization height on the VAWT performance has been studied for a three-straight-bladed VAWT. The results have demonstrated that the GF addition of an optimum height (3\% of $c$ ) can increase the power coefficient by around $80 \%$ compared to original blade geometry. The rotation has small impact on optimum GF height compared to a stationary single airfoil (i.e. $2 \%$ of $c$ ). The flow characteristics indicate that the GF addition can delay the deep stall of the turbine blades, and also it can produce positive moment coefficients, thus having improved power coefficient.

\section{Acknowledgments}

The first author would like to acknowledge the Ministry of Research, Technology and Higher Education of Indonesia and Indonesian Endowment Fund for Education (LPDP) of the Ministry of Finance of Indonesia for the financial support through BUDI LPDP scholarship.

\section{References}

1. J. O. Dabiri, J. Renew. Sustain. Energy, 3 (2011) p. 043104.

2. R. H. Liebeck, J. Aircraft, 15 (1978) pp. 547-561.

3. M. Mohammadi, A. Doosttalab and M. Doosttalab, ASME 2012 Early Career Tech. J., 11 (2012) pp 244-250.

4. W. Zhao, J. Zhu and P. Liu, in Proc. Asia-Pacific Power and Energy Engineering Conference, Shanghai, China, pp. 1-4, 2012.

5. H. Zhu, et al., J. Wind Eng. Ind. Aerod., 186 (2019) pp. 17-31.

6. A. Rezaeiha, H. Montazeri and B. Blocken, Energy Convers. Manag., 169 (2018) pp. 45-77.

7. I. Aramendia, et al., in Proc. The 2nd International Research Conference on Sustainable Energy, Engineering, Materials and Environment, Mieres, Spain, 2 (23) 1448, 2018.

8. M. R. Castelli, A. Englaro, and E. Benini, Energy, 36 (2011) pp. 4919-4934. 